\title{
Phytoprotection
}

\section{Ice nucleation activity identified in some phytopathogenic Fusarium species}

\author{
C. Richard, J.-G. Martin et S. Pouleur
}

Volume 77, numéro 2, 1996

URI : https://id.erudit.org/iderudit/706104ar

DOI : https://doi.org/10.7202/706104ar

Aller au sommaire du numéro

Éditeur(s)

Société de protection des plantes du Québec (SPPQ)l

ISSN

0031-9511 (imprimé)

1710-1603 (numérique)

Découvrir la revue

Citer cet article

Richard, C., Martin, J.-G. \& Pouleur, S. (1996). Ice nucleation activity identified in some phytopathogenic Fusarium species. Phytoprotection, 77(2), 83-92. https://doi.org/10.7202/706104ar
Résumé de l'article

Afin de mieux connaître les facteurs qui influencent leur pouvoir pathogène, nous avons recherché l'activité glaçogène chez trois espèces de Fusarium : le Fusarium oxysporum, le $F$ sporotrichioides et le $F$ tricinctum. Le $F$ acuminatum et le $F$ avenaceum ont servi de témoins positifs (lna+). La recherche de l'activité glaçogène a été effectuée à l'aide d'un test rapide et simple de nucleation en éprouvettes. Douze des 42 isolats testés du $F$ oxysporum et 8 des 14 isolats testés du $F$ tricinctum se sont révélés positifs alors que les isolats du $F$ sporotrichioides furent négatifs. La température de nucleation tendait à augmenter avec le temps, pouvant atteindre $-1^{\circ} \mathrm{C}$, température maximale rapportée pour les bactéries et beaucoup plus élevée que la température maximale connue pour les champignons et pour les algues et les cyanobactéries de lichen en culture pure. C'est la première fois qu'une activité glaçogène est démontrée pour le $F$ oxysporum. 


\title{
Communication brève / Short Communication
}

\section{Ice nucleation activity identified in some phytopathogenic Fusarium species}

\author{
Claude Richard', Jean-Guy Martin', and Stéphan Pouleur²
}

Received 1996-03-22; accepted 1996-09-02

In order to know which species of Fusarium are ice nucleating and to determine the factors affecting their pathogenicity, ice nucleation activity (INA) was examined in Fusarium oxysporum, F. sporotrichioides, and F. tricinctum. Positive controls (Ina ${ }^{+}$) used were $F$. acuminatum and $F$. avenaceum. The test for fungal INA was done with a simple and rapid tube nucleation assay. Twelve out of the $42 \mathrm{~F}$. oxysporum isolates, and 8 out of $14 \mathrm{~F}$. tricinctum isolates were Ina+. No INA was detected in F. sporotrichioides. In this test, the threshold freezing temperature tended to increase with culture age, reaching a peak of $-1^{\circ} \mathrm{C}$ in a few samples, which is as high as the warmest INA reported for bacteria, and higher than the INA detected in pure cultures of free-living fungi, lichen fungi, lichen algae and cyanobacteria. This is the first report of INA for F. oxysporum.

Richard, C., J.-G. Martin, and S. Pouleur. 1996. Ice nucleation activity identified in some phytopathogenic Fusarium species. PHYTOPROTECTION 77 : 83-92.

Afin de mieux connaître les facteurs qui influencent leur pouvoir pathogène, nous avons recherché l'activité glaçogène chez trois espèces de Fusarium : le Fusarium oxysporum, le $F$. sporotrichioides et le $F$. tricinctum. Le F. acuminatum et le $F$. avenaceum ont servi de témoins positifs $($ Ina+). La recherche de l'activité glaçogène a été effectuée à l'aide d'un test rapide et simple de nucléation en éprouvettes. Douze des 42 isolats testés du $F$. oxysporum et 8 des 14 isolats testés du F. tricinctum se sont révélés positifs alors que les isolats du $F$. sporotrichioides furent négatifs. La température de nucléation tendait à augmenter avec le temps, pouvant atteindre $-1^{\circ} \mathrm{C}$, température maximale rapportée pour les bactéries et beaucoup plus élevée que la température maximale connue pour les champignons et pour les algues et les cyanobactéries de lichen en culture pure. C'est la première fois qu'une activité glaçogène est démontrée pour le $F$. oxysporum.

Ice nucleation is the initiation of the crystallization of subcooled water (below $0^{\circ} \mathrm{C}$ ). Ice nucleation activity (INA) at temperatures above $-5^{\circ} \mathrm{C}$ has been known in bacteria since 1976 (Vali et al. 1976). Recently, INA was associated with lichens (Kieft 1988) and with free-living fungi (Pouleur et al. 1992; Tsumuki et al. 1992).
Until now, non-lichen fungal INA has been
known only in the genus Fusarium (Ash-
worth and Kieft 1995). Two species of
Fusarium have been reported as ice-nu-
cleating (Ina+) fungi : F. acuminatum Ellis
\& Everh. (Pouleur et al. 1992) and
F. avenaceum (Fr.: Fr.) Sacc. (Hasegawa
et al. 1994; Pouleur et al. 1992; Sychrova

1. Soils and Crops Research and Development Centre, Agriculture and Agri-Food Canada, 2560 Hochelaga Blvd., Sainte-Foy, Quebec, Canada G1V 2J3. Contribution No. 530.

2. Département de phytologie, Université Laval, Québec, Canada G1K 7P4 
et al. 1994). INA initiated at relatively high temperature by these pathogenic fungi may give them an advantage for root invasion. Richard et al. $(1982,1985)$ and Smith and Olien (1978) showed that infection of plant roots by fusaria is enhanced by freezing. It is thus interesting to know which species of Fusarium are Ina ${ }^{+}$in order to better understand the factors affecting their pathogenicity and to check the validity of this criteria for use in the identification of Ina ${ }^{+}$Fusarium species.

INA was investigated in three species of Fusarium, which are well known plant pathogens and common soil inhabitants (Farr et al. 1989) : F. oxysporum Schlechtend.: Fr. (42 isolates), F. sporotrichioides Sherb. (14 isolates) and F. tricinctum (Corda) Sacc. (14 isolates). Two isolates of both F. acuminatum and $F$. avenaceum were used as Ina ${ }^{+}$ controls. Two cultures (replicates) of each isolate were tested. The test for fungal INA was done by using a tube nucleation test (Pouleur et al. 1992). Instead of scraping off the mycelial mat from the surface of the agar, a mycelium plug ( $5 \mathrm{~mm}$ diam) was recovered from the oldest part of the colony (near the centre). The plug was dropped in a small test tube $(13 \times 100 \mathrm{~mm})$ containing $2 \mathrm{~mL}$ sterile deionized water, subcooled at $-5.0^{\circ} \mathrm{C}$ in a Lauda refrigerating circulator bath (model RC-6; Brinkman Instrument Co., Rexdale, Ontario, Canada) and free of nuclei according to a test at $-10.0^{\circ} \mathrm{C}$ for $1 \mathrm{~h}$. Freezing of the tube contents within 10 min of dropping the plug was recorded as positive $\left(\right.$ Ina $\left.^{+}\right)$for that species. The cultures were tested after 7, 14, 21 and $28 \mathrm{~d}$ of growth. Following all the nucleation tests, the tubes and their contents were allowed to warm to room temperature. After $20 \mathrm{~min}$, they were put back in the bath for 20 min and cooled down to $0^{\circ} \mathrm{C}$. Afterwards the temperature was lowered at the rate of $0.1^{\circ} \mathrm{C} \mathrm{min}-1$ until it reached $-10.0^{\circ} \mathrm{C}$. The temperature at which the contents of the tubes froze (the threshold temperature) was recorded.

After $7 \mathrm{~d}$, and up to $28 \mathrm{~d}$, both isolates of $F$. acuminatum and $F$. avenaceum, the Ina ${ }^{+}$controls, were positive (Table 1 ). Out of 43 F. oxysporum isolates, 12 were Ina+. Isolates nos. 301, 709, 710 and 712 were the most consistent over time and replicates, and may definitively be regarded as Ina+. The other ones did not react consistently. It is not surprising that some strains of an Ina ${ }^{+}$species were Ina; in bacteria, not all strains within a species are Ina ${ }^{+}$(Hirano et al. 1978; Paulin and Luisetti 1978). In a previous survey of INA in Fusarium species, no Ina ${ }^{+}$isolates of F. oxysporum had been found (Pouleur et al. 1992).

No INA was detected in F. sporotrichioides except in two instances: at $7 \mathrm{~d}$ for isolate 538 sample $B$ and at $21 \mathrm{~d}$ for isolate 609 sample $B$. The observed threshold temperatures, $-8.2^{\circ} \mathrm{C}$ and $-10^{\circ} \mathrm{C}$ for 538 and $609 \mathrm{~B}$, respectively, are too low to be considered Ina+.

Of the $14 \mathrm{~F}$. tricinctum isolates tested, 9 were Ina+; 7 of these were consistently Ina ${ }^{+}$. Isolates 663 and 689 were positive at 14 and $21 \mathrm{~d}$ respectively, but did not exhibit a threshold temperature higher than $-5.5^{\circ} \mathrm{C}$. The maximum temperature at which INA was reported for F. tricinctum was $-5.6^{\circ} \mathrm{C}$ (Sychrova et al. 1994). This is not recognized as Type 1 INA or warm INA temperature, i.e. nuclei active at temperatures above $-5.0^{\circ} \mathrm{C}$ (Phelps et al. 1986). The nucleation temperatures of the Ina ${ }^{+}$isolates of $F$. tricinctum in our test were usually higher than $-5.0^{\circ} \mathrm{C}$ with a peak at $-1.0^{\circ} \mathrm{C}$ at $21 \mathrm{~d}$ for isolate $666 \mathrm{~A}$. This confirms that $F$. tricinctum is Ina $a^{+}$and indicates that it probably produces Type I nuclei.

When an Ina+ isolate was consistent over time and replicates, it usually showed a positive reaction as soon as $7 \mathrm{~d}$ and at all sampling periods. The threshold temperature tended to increase with tirne in most Ina ${ }^{+}$isolates (Nos. 116, 368, 250, $301,709,710,662,665,666,684,685,686$ 687 and 689 ), reaching a peak of $-1^{\circ} \mathrm{C}$ in some instances. This temperature is as high as the warmest INA reported for bacteria, and higher than the INA detected in pure cultures of free-living fungi, lichen fungi, lichen algae and cyanobacteria as compiled by Ashworth and Kieft (1995). The highest INA previously reported is $-1.9^{\circ} \mathrm{C}$ for the lichen mycobiont Rhizoplaca chrysoleuca (Sm.) Zopf. In some other Ina ${ }^{+}$isolates, the threshold temperature fluctuated. 
Table 1. Ice nucleation activity (INA) of five Fusarium species, and ice nucleation threshold temperatures at four different ages

\begin{tabular}{|c|c|c|c|c|c|c|c|c|c|c|c|}
\hline \multirow{2}{*}{$\begin{array}{l}\text { SRSF } \\
\text { No. }^{a}\end{array}$} & \multirow[b]{2}{*}{ Host } & \multirow[b]{2}{*}{ Location } & \multirow[b]{2}{*}{ Repl. } & \multicolumn{4}{|c|}{ INA $^{\mathrm{b}}$} & \multicolumn{4}{|c|}{$\begin{array}{c}\text { Threshold temperatures }{ }^{c} \\
\left({ }^{\circ} \mathrm{C}\right)\end{array}$} \\
\hline & & & & $7 \mathrm{~d}$ & $14 \mathrm{~d}$ & $21 \mathrm{~d}$ & $28 \mathrm{~d}$ & $7 \mathrm{~d}$ & $14 \mathrm{~d}$ & $21 \mathrm{~d}$ & $28 \mathrm{~d}$ \\
\hline \multicolumn{12}{|c|}{ Fusarium acuminatum (control) } \\
\hline 116 & Medicago sativa $\mathrm{L}$. & Pennsylvania, USA & $\begin{array}{l}\text { A } \\
B\end{array}$ & $\begin{array}{l}+ \\
+\end{array}$ & $\begin{array}{l}+ \\
+\end{array}$ & $\begin{array}{l}+ \\
+\end{array}$ & $\begin{array}{l}+ \\
+\end{array}$ & $\begin{array}{l}-5.1 \\
-4.6\end{array}$ & $\begin{array}{l}-3.8 \\
-3.9\end{array}$ & $\begin{array}{l}-3.8 \\
-3.6\end{array}$ & $\begin{array}{l}-3.5 \\
-4.4\end{array}$ \\
\hline 368 & Pisum sativum $\mathrm{L}$. & Quebec, Canada & $\begin{array}{l}\text { A } \\
B\end{array}$ & $\begin{array}{l}+ \\
+\end{array}$ & $\begin{array}{l}+ \\
+\end{array}$ & $\begin{array}{l}+ \\
+\end{array}$ & $\begin{array}{l}+ \\
+\end{array}$ & $\begin{array}{l}-4.8 \\
-4.6\end{array}$ & $\begin{array}{l}-4.1 \\
-3.9\end{array}$ & $\begin{array}{l}-4.3 \\
-3.5\end{array}$ & $\begin{array}{l}-4.5 \\
-4.3\end{array}$ \\
\hline \multicolumn{12}{|c|}{ Fusarium avenaceum (control) } \\
\hline 250 & Medicago sativa L. & Quebec, Canada & $\begin{array}{l}A \\
B\end{array}$ & $\begin{array}{l}+ \\
+\end{array}$ & $\begin{array}{l}+ \\
+\end{array}$ & $\begin{array}{l}+ \\
+\end{array}$ & $\begin{array}{l}+ \\
+\end{array}$ & $\begin{array}{l}-5.3 \\
-4.3\end{array}$ & $\begin{array}{l}-3.8 \\
-3.9\end{array}$ & $\begin{array}{l}-3.7 \\
-4.2\end{array}$ & $\begin{array}{l}-3.9 \\
-4.0\end{array}$ \\
\hline 411 & Medicago sativa $\mathrm{L}$. & Quebec, Canada & $\begin{array}{l}\text { A } \\
B\end{array}$ & $\begin{array}{l}+ \\
+\end{array}$ & $\begin{array}{l}+ \\
+\end{array}$ & $\begin{array}{l}+ \\
+\end{array}$ & $\begin{array}{l}+ \\
+\end{array}$ & $\begin{array}{l}-3.8 \\
-3.8\end{array}$ & $\begin{array}{l}-3.5 \\
-2.9\end{array}$ & $\begin{array}{l}-3.6 \\
-3.6\end{array}$ & $\begin{array}{l}-3.4 \\
-4.0\end{array}$ \\
\hline \multicolumn{12}{|c|}{ Fusarium oxysporum } \\
\hline 39 & Unknown & Manitoba, Canada & $\begin{array}{l}A \\
B\end{array}$ & - & - & - & - & $\frac{-9.2}{-}$ & $\underline{-}^{d}$ & - & $\overline{-9.4}$ \\
\hline 41 & Trifolium pratense $\mathrm{L}$. & Quebec, Canada & $\begin{array}{l}A \\
B\end{array}$ & - & - & - & - & - & $\overline{-8.1}$ & - & - \\
\hline 114 & Medicago sativa $\mathrm{L}$. & Pennsylvania, USA & $\begin{array}{l}\text { A } \\
B\end{array}$ & - & - & - & - & $\begin{array}{l}-9.8 \\
-9.7\end{array}$ & - & - & - \\
\hline 115 & Medicago sativa $\mathrm{L}$. & Maryland, USA & $\begin{array}{l}A \\
B\end{array}$ & - & - & - & - & - & - & - & $\overline{-8.9}$ \\
\hline 117 & Medicago sativa L. & Maryland, USA & $\begin{array}{l}A \\
B\end{array}$ & - & - & - & - & - & - & - & $\overline{-9.8}$ \\
\hline 120 & Medicago sativa $\mathrm{L}$. & Maryland, USA & $\begin{array}{l}A \\
B\end{array}$ & - & - & - & - & - & - & $\overline{-7.5}$ & - \\
\hline 242 & Medicago sativa L. & Quebec, Canada & $\begin{array}{l}A \\
B\end{array}$ & - & - & - & - & $\begin{array}{c}-7.5 \\
-\end{array}$ & - & - & - \\
\hline
\end{tabular}




\section{PHYTOPROTECTION 77 (2) 1996}

Table 1. Ice nucleation activity (INA) of five Fusarium species, and ice nucleation threshold temperatures at four different ages (suite)

\begin{tabular}{|c|c|c|c|c|c|c|c|c|c|c|c|}
\hline \multirow{2}{*}{$\begin{array}{l}\text { SRSF } \\
\text { No. }^{a}\end{array}$} & \multirow[b]{2}{*}{ Host } & \multirow[b]{2}{*}{ Location } & \multirow[b]{2}{*}{ Repl. } & \multicolumn{4}{|c|}{ INA $^{\mathrm{b}}$} & \multicolumn{4}{|c|}{$\begin{array}{c}\text { Threshold temperatures }{ }^{\mathrm{c}} \\
\left({ }^{\circ} \mathrm{C}\right)\end{array}$} \\
\hline & & & & $7 \mathrm{~d}$ & $14 \mathrm{~d}$ & $21 \mathrm{~d}$ & $28 \mathrm{~d}$ & $\overline{7 d}$ & $14 \mathrm{~d}$ & $21 \mathrm{~d}$ & $28 \mathrm{~d}$ \\
\hline 245 & Medicago sativa L. & Quebec, Canada & $\begin{array}{l}\text { A } \\
B\end{array}$ & - & - & - & - & - & - & $\overline{-5.5}$ & - \\
\hline 248 & Medicago sativa $\mathrm{L}$. & Quebec, Canada & $\begin{array}{l}\text { A } \\
B\end{array}$ & - & - & - & - & - & - & $\overline{-9.4}$ & - \\
\hline 249 & Medicago sativa $\mathrm{L}$. & Quebec, Canada & $\begin{array}{l}\text { A } \\
B\end{array}$ & - & - & - & - & - & $\begin{array}{l}-9.3 \\
-9.1\end{array}$ & $\begin{array}{l}-6.7 \\
-5.9\end{array}$ & $\begin{array}{l}-8.5 \\
-8.9\end{array}$ \\
\hline 252 & Medicago sativa $\mathrm{L}$. & Quebec, Canada & $\begin{array}{l}\text { A } \\
B\end{array}$ & - & - & - & - & - & - & - & - \\
\hline 260 & Medicago sativa $\mathrm{L}$. & Quebec, Canada & $\begin{array}{l}A \\
B\end{array}$ & - & - & - & - & $\begin{array}{c}-5.7 \\
-\end{array}$ & - & - & $\begin{array}{c}-9.2 \\
-\end{array}$ \\
\hline 296 & Medicago sativa $\mathrm{L}$. & Quebec, Canada & $\begin{array}{l}A \\
B\end{array}$ & - & - & $\begin{array}{l}- \\
-\end{array}$ & - & - & - & - & - \\
\hline 300 & Medicago sativa $\mathrm{L}$. & Quebec, Canada & $\begin{array}{l}\text { A } \\
B\end{array}$ & - & - & - & - & - & - & - & - \\
\hline 301 & Vicia faba L. & Quebec, Canada & $\begin{array}{l}A \\
B\end{array}$ & - & $\begin{array}{l}+ \\
+\end{array}$ & $\begin{array}{l}+ \\
+\end{array}$ & $\begin{array}{l}+ \\
+\end{array}$ & $\overline{-9.8}$ & $\begin{array}{l}-3.0 \\
-2.8\end{array}$ & $\begin{array}{l}-2.8 \\
-3.7\end{array}$ & $\begin{array}{l}-2.5 \\
-1.0\end{array}$ \\
\hline 304 & Solanum tuberosum L. & Quebec, Canada & $\begin{array}{l}A \\
B\end{array}$ & - & - & - & - & $\begin{array}{c}-7.4 \\
-\end{array}$ & - & $\stackrel{-5.2}{-}$ & - \\
\hline 314 & Medicago sativa $\mathrm{L}$. & Quebec, Canada & $\begin{array}{l}A \\
B\end{array}$ & - & - & - & - & - & - & - & - \\
\hline 322 & Medicago sativa $\mathrm{L}$. & Quebec, Canada & $\begin{array}{l}A \\
B\end{array}$ & - & - & - & - & - & $\overline{-7.9}$ & $\stackrel{-5.6}{-}$ & - \\
\hline 334 & Medicago sativa L. & Quebec, Canada & $\begin{array}{l}\text { A } \\
B\end{array}$ & + & - & $\begin{array}{l}- \\
+\end{array}$ & - & - & - & - & - \\
\hline 336 & Phaseolus vulgaris L. & Quebec, Canada & $\begin{array}{l}A \\
B\end{array}$ & - & - & $\begin{array}{l}+ \\
+\end{array}$ & - & $\overline{-9.6}$ & $\overline{-9.2}$ & $\begin{array}{l}-6.7 \\
-5.7\end{array}$ & $\overline{-8.3}$ \\
\hline
\end{tabular}




\begin{tabular}{|c|c|c|c|c|c|c|c|c|c|c|c|}
\hline \multirow{2}{*}{$\begin{array}{l}\text { SRSF } \\
\text { No. }^{a}\end{array}$} & \multirow[b]{2}{*}{ Host } & \multirow[b]{2}{*}{ Location } & \multirow[b]{2}{*}{ Repl. } & \multicolumn{4}{|c|}{ INA $^{\mathrm{b}}$} & \multicolumn{4}{|c|}{$\begin{array}{l}\text { Threshold temperatures } \\
\qquad\left({ }^{\circ} \mathrm{C}\right)\end{array}$} \\
\hline & & & & $\overline{7 d}$ & $14 \mathrm{~d}$ & $21 \mathrm{~d}$ & $28 \mathrm{~d}$ & $\overline{7 d}$ & $14 \mathrm{~d}$ & $21 d$ & $28 d$ \\
\hline 337 & Phaseolus vulgaris L. & Alberta, Canada & $\begin{array}{l}A \\
B\end{array}$ & $\begin{array}{l}- \\
-\end{array}$ & - & $\begin{array}{l}- \\
-\end{array}$ & $\begin{array}{l}- \\
-\end{array}$ & - & - & - & - \\
\hline 338 & Phaseolus vulgaris L. & Alberta, Canada & $\begin{array}{l}A \\
B\end{array}$ & $\begin{array}{l}- \\
-\end{array}$ & $\begin{array}{l}- \\
-\end{array}$ & $\begin{array}{l}+ \\
-\end{array}$ & $\begin{array}{l}- \\
-\end{array}$ & - & - & - & - \\
\hline 340 & Pisum sativum $\mathrm{L}$. & Alberta, Canada & $\begin{array}{l}\mathrm{A} \\
\mathrm{B}\end{array}$ & $\begin{array}{l}- \\
-\end{array}$ & $\begin{array}{l}- \\
-\end{array}$ & $\begin{array}{l}- \\
-\end{array}$ & - & - & - & - & - \\
\hline 352 & Pisum sativum L. & Quebec, Canada & $\begin{array}{l}\mathrm{A} \\
\mathrm{B}\end{array}$ & $\begin{array}{l}- \\
-\end{array}$ & - & $\begin{array}{l}- \\
-\end{array}$ & $\begin{array}{l}- \\
+\end{array}$ & -8.3 & - & $\overline{-3.6}$ & $\overline{-5.7}$ \\
\hline 359 & Pisum sativum L. & Quebec, Canada & $\begin{array}{l}A \\
B\end{array}$ & $\begin{array}{l}- \\
-\end{array}$ & $\begin{array}{l}- \\
-\end{array}$ & - & - & $\begin{array}{l}-9.1 \\
-7.5\end{array}$ & $\overline{-6.4}$ & $\begin{array}{l}-7.9 \\
-\end{array}$ & - \\
\hline 360 & Pisum sativum L. & Quebec, Canada & $\begin{array}{l}A \\
B\end{array}$ & $\begin{array}{l}- \\
-\end{array}$ & - & - & $\begin{array}{l}- \\
-\end{array}$ & - & $-\overline{10.0}$ & - & - \\
\hline 376 & Phaseolus vulgaris $\mathrm{L}$. & Quebec, Canada & $\begin{array}{l}A \\
B\end{array}$ & $\begin{array}{l}- \\
-\end{array}$ & $\begin{array}{l}- \\
-\end{array}$ & $\begin{array}{l}- \\
-\end{array}$ & - & - & - & - & - \\
\hline 398 & Medicago sativa L. & Quebec, Canada & $\begin{array}{l}A \\
B\end{array}$ & $\begin{array}{l}- \\
-\end{array}$ & $\begin{array}{l}- \\
-\end{array}$ & $\begin{array}{l}- \\
-\end{array}$ & $\begin{array}{l}- \\
+\end{array}$ & - & - & - & $\overline{-9.0}$ \\
\hline 527 & Lupinus albus L. & Quebec, Canada & $\begin{array}{l}A \\
B\end{array}$ & $\begin{array}{l}- \\
-\end{array}$ & $\begin{array}{l}- \\
-\end{array}$ & $\begin{array}{l}- \\
-\end{array}$ & $\begin{array}{l}- \\
-\end{array}$ & - & - & - & - \\
\hline 528 & Lupinus albus L. & Quebec, Canada & $\begin{array}{l}A \\
B\end{array}$ & $\begin{array}{l}- \\
-\end{array}$ & $\begin{array}{l}- \\
-\end{array}$ & - & $\begin{array}{l}- \\
-\end{array}$ & - & $\overline{-7.4}$ & $-\overline{10.0}$ & - \\
\hline 529 & Lupinus albus L. & Quebec, Canada & $\begin{array}{l}\text { A } \\
B\end{array}$ & $\begin{array}{l}- \\
-\end{array}$ & $\begin{array}{l}- \\
-\end{array}$ & $\begin{array}{l}- \\
-\end{array}$ & $\begin{array}{l}- \\
-\end{array}$ & - & - & - & - \\
\hline 573 & Mangifera indica L. & Malaysia & $\begin{array}{l}A \\
B\end{array}$ & $\begin{array}{l}- \\
-\end{array}$ & $\begin{array}{l}- \\
-\end{array}$ & - & $\begin{array}{l}- \\
-\end{array}$ & $\overline{-9.4}$ & - & - & -8.3 \\
\hline 574 & Insects & Malaysia & $\begin{array}{l}A \\
B\end{array}$ & - & - & - & - & - & - & $-\overline{10.0}$ & - \\
\hline 575 & Cucumis melo L. & Malaysia & $\begin{array}{l}A \\
B\end{array}$ & - & - & - & - & - & - & - & - \\
\hline
\end{tabular}


PHYTOPROTECTION 77 (2) 1996

Table 1. Ice nucleation activity (INA) of five Fusarium species, and ice nucleation threshold temperatures at four different ages (suite)

\begin{tabular}{|c|c|c|c|c|c|c|c|c|c|c|c|}
\hline \multirow{2}{*}{$\begin{array}{l}\text { SRSF } \\
\text { No. }^{\text {a }}\end{array}$} & \multirow[b]{2}{*}{ Host } & \multirow[b]{2}{*}{ Location } & \multirow[b]{2}{*}{ Repl. } & \multicolumn{4}{|c|}{ INA $^{\mathrm{b}}$} & \multicolumn{4}{|c|}{$\begin{array}{c}\text { Threshold temperatures }{ }^{\mathrm{c}} \\
\left({ }^{\circ} \mathrm{C}\right)\end{array}$} \\
\hline & & & & $\overline{7 d}$ & $14 \mathrm{~d}$ & $21 \mathrm{~d}$ & $28 \mathrm{~d}$ & $7 \mathrm{~d}$ & $14 \mathrm{~d}$ & $21 \mathrm{~d}$ & $28 \mathrm{~d}$ \\
\hline 576 & Asparagus officinalis L. & Malaysia & $\begin{array}{l}\text { A } \\
B\end{array}$ & - & + & + & $\begin{array}{l}- \\
-\end{array}$ & - & -3.6 & - & - \\
\hline 596 & Lab. contamination & Quebec, Canada & $\begin{array}{l}\text { A } \\
B\end{array}$ & $\begin{array}{l}- \\
-\end{array}$ & - & - & + & -5.5 & - & -9.7 & -7.2 \\
\hline 676 & Unknown & Unknown & $\begin{array}{l}\text { A } \\
B\end{array}$ & - & - & - & - & - & - & - & - \\
\hline 708 & Medicago sativa $\mathrm{L}$. & Quebec, Canada & $\begin{array}{l}A \\
B\end{array}$ & - & $\begin{array}{l}- \\
-\end{array}$ & $\begin{array}{l}- \\
-\end{array}$ & + & - & $\overline{-9.8}$ & - & - \\
\hline 709 & Medicago sativa $\mathrm{L}$. & Quebec, Canada & $\begin{array}{l}A \\
B\end{array}$ & $\begin{array}{l}- \\
+\end{array}$ & $\begin{array}{l}+ \\
+\end{array}$ & $\begin{array}{l}+ \\
+\end{array}$ & $\begin{array}{l}+ \\
+\end{array}$ & $\begin{array}{l}-6.0 \\
-3.3\end{array}$ & $\begin{array}{l}-1.8 \\
-1.4\end{array}$ & $\begin{array}{l}-3.8 \\
-1.5\end{array}$ & $\begin{array}{l}-1.0 \\
-1.0\end{array}$ \\
\hline 710 & Lab. contamination & Quebec, Canada & $\begin{array}{l}A \\
B\end{array}$ & $\begin{array}{l}+ \\
-\end{array}$ & $\begin{array}{l}+ \\
+\end{array}$ & + & $\begin{array}{l}+ \\
+\end{array}$ & - & $\begin{array}{l}-3.6 \\
-4.0\end{array}$ & $\begin{array}{c}-2.5 \\
-\end{array}$ & $\begin{array}{l}-1.2 \\
-2.0\end{array}$ \\
\hline 711 & Medicago sativa L. & Ontario, Canada & $\begin{array}{l}A \\
B\end{array}$ & - & - & - & - & - & $\begin{array}{l}-7.1 \\
-\end{array}$ & - & $\overline{-9.9}$ \\
\hline 712 & Medicago sativa L. & Ontario, Canada & $\begin{array}{l}A \\
B\end{array}$ & - & - & $\begin{array}{l}+ \\
+\end{array}$ & 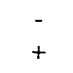 & - & $\begin{array}{c}-4.0 \\
-\end{array}$ & $\overline{-3.7}$ & $\overline{-2.6}$ \\
\hline \multicolumn{12}{|c|}{ Fusarium sporotrichioides } \\
\hline 538 & Zea mais $\mathrm{L}$. & Ontario, Canada & $\begin{array}{l}A \\
B\end{array}$ & $\begin{array}{l}- \\
+\end{array}$ & - & - & - & $\overline{-8.2}$ & $\frac{-6.7}{-}$ & $\begin{array}{l}-7.8 \\
-\end{array}$ & - \\
\hline 607 & Hay & Saskatchewan, Canada & $\begin{array}{l}\text { A } \\
\text { B }\end{array}$ & - & - & - & - & $\begin{array}{c}-9.6 \\
-\end{array}$ & - & $\overline{-9.3}$ & - \\
\hline 608 & Saccharum officinarum L. & Guyana & $\begin{array}{l}A \\
B\end{array}$ & - & - & - & - & $\begin{array}{c}-7.4 \\
-\end{array}$ & - & $\overline{-8.9}$ & $\begin{array}{c}-- \\
-6.1\end{array}$ \\
\hline 609 & Turf & Alberta, Canada & $\begin{array}{l}A \\
B\end{array}$ & - & - & $\begin{array}{l}- \\
+\end{array}$ & - & $\begin{array}{c}-9.3 \\
-\end{array}$ & $\begin{array}{c}-9.7 \\
-\end{array}$ & - & - \\
\hline
\end{tabular}




\begin{tabular}{|c|c|c|c|c|c|c|c|c|c|c|c|}
\hline \multirow{2}{*}{$\begin{array}{l}\text { SRSF } \\
\text { No. }^{a}\end{array}$} & \multirow[b]{2}{*}{ Host } & \multirow[b]{2}{*}{ Location } & \multirow[b]{2}{*}{ Repl. } & \multicolumn{4}{|c|}{ INA $^{\mathrm{b}}$} & \multicolumn{4}{|c|}{$\begin{array}{c}\text { Threshold temperatures }{ }^{\mathrm{c}} \\
\left({ }^{\circ} \mathrm{C}\right)\end{array}$} \\
\hline & & & & $\overline{7 d}$ & $14 \mathrm{~d}$ & $21 \mathrm{~d}$ & $28 \mathrm{~d}$ & $7 \mathrm{~d}$ & $14 \mathrm{~d}$ & $21 \mathrm{~d}$ & $28 \mathrm{~d}$ \\
\hline 679 & Unknown & Unknown & $\begin{array}{l}A \\
B\end{array}$ & - & $\begin{array}{l}- \\
-\end{array}$ & - & - & $\overline{-9.7}$ & - & - & - \\
\hline 692 & Unknown & Unknown & $\begin{array}{l}A \\
B\end{array}$ & - & - & - & - & $\overline{-9.4}$ & - & $\begin{array}{c}-7.0 \\
-\end{array}$ & - \\
\hline 693 & Unknown & Unknown & $\begin{array}{l}A \\
B\end{array}$ & $\begin{array}{l}- \\
-\end{array}$ & - & - & $\begin{array}{l}- \\
-\end{array}$ & - & - & - & - \\
\hline 694 & Unknown & Unknown & $\begin{array}{l}A \\
B\end{array}$ & - & - & - & - & - & - & - & - \\
\hline 695 & Unknown & Unknown & $\begin{array}{l}\text { A } \\
B\end{array}$ & $\begin{array}{l}- \\
-\end{array}$ & - & $\begin{array}{l}- \\
-\end{array}$ & $\begin{array}{l}- \\
-\end{array}$ & $\overline{-9.5}$ & - & - & - \\
\hline 696 & Unknown & Unknown & $\begin{array}{l}A \\
B\end{array}$ & - & - & - & - & $\overline{-7.7}$ & - & - & - \\
\hline 697 & Unknown & Unknown & $\begin{array}{l}A \\
B\end{array}$ & - & - & - & - & - & - & - & - \\
\hline 698 & Unknown & Unknown & $\begin{array}{l}\text { A } \\
B\end{array}$ & $\begin{array}{l}- \\
-\end{array}$ & - & - & $\begin{array}{l}- \\
-\end{array}$ & - & $\overline{-7.8}$ & $\overline{-9.1}$ & - \\
\hline 699 & Unknown & Unknown & $\begin{array}{l}A \\
B\end{array}$ & - & - & - & - & $\overline{-7.4}$ & - & - & - \\
\hline 700 & Unknown & Unknown & $\begin{array}{l}\text { A } \\
B\end{array}$ & - & - & - & - & - & - & - & - \\
\hline \multicolumn{12}{|c|}{ Fusarium tricinctum } \\
\hline 121 & Medicago sativa L. & Pennsylvania, USA & $\begin{array}{l}A \\
B\end{array}$ & - & - & - & - & - & $\overline{-8.8}$ & $\overline{-9.8}$ & - \\
\hline 612 & Brassica napus L. & Alberta, Canada & $\begin{array}{l}A \\
B\end{array}$ & $\begin{array}{l}+ \\
+\end{array}$ & $\begin{array}{l}+ \\
+\end{array}$ & $\begin{array}{l}+ \\
+\end{array}$ & $\begin{array}{l}+ \\
+\end{array}$ & $\begin{array}{l}-3.9 \\
-4.0\end{array}$ & $\begin{array}{l}-3.6 \\
-3.9\end{array}$ & $\begin{array}{l}-3.7 \\
-2.6\end{array}$ & $\begin{array}{l}-3.5 \\
-3.9\end{array}$ \\
\hline
\end{tabular}


PHYTOPROTECTION 77 (2) 1996

Table 1. Ice nucleation activity (INA) of five Fusarium species, and ice nucleation threshold temperatures at four different ages (suite)

\begin{tabular}{|c|c|c|c|c|c|c|c|c|c|c|c|}
\hline \multirow{2}{*}{$\begin{array}{l}\text { SRSF } \\
\text { No. }^{a}\end{array}$} & \multirow[b]{2}{*}{ Host } & \multirow[b]{2}{*}{ Location } & \multirow[b]{2}{*}{ Repl. } & \multicolumn{4}{|c|}{ INA $^{\mathrm{b}}$} & \multicolumn{4}{|c|}{$\begin{array}{c}\text { Threshold temperatures }{ }^{c} \\
\left({ }^{\circ} \mathrm{C}\right) \\
\end{array}$} \\
\hline & & & & $\overline{7 d}$ & $14 \mathrm{~d}$ & $21 \mathrm{~d}$ & $28 d$ & $\overline{7 d}$ & $14 \mathrm{~d}$ & $21 d$ & $28 \mathrm{~d}$ \\
\hline 663 & Hordeum vulgare L. & Poland & $\begin{array}{l}A \\
B\end{array}$ & - & $\begin{array}{l}+ \\
-\end{array}$ & - & - & $\overline{-7.4}$ & $\begin{array}{l}-5.5 \\
-8.3\end{array}$ & $\begin{array}{l}-9.5 \\
-8.2\end{array}$ & $\begin{array}{l}-9.0 \\
-9.2\end{array}$ \\
\hline 664 & Triticum aestivum L. & Poland & $\begin{array}{l}\mathrm{A} \\
\mathrm{B}\end{array}$ & - & $\begin{array}{l}- \\
-\end{array}$ & - & $\begin{array}{l}- \\
-\end{array}$ & - & - & $\overline{-}$ & - \\
\hline 665 & Hordeum vulgare $\mathrm{L}$. & Poland & $\begin{array}{l}A \\
B\end{array}$ & $\begin{array}{l}- \\
+\end{array}$ & $\begin{array}{l}+ \\
+\end{array}$ & $\dot{-}+$ & $\begin{array}{l}+ \\
+\end{array}$ & $\begin{array}{l}-6.4 \\
-5.7\end{array}$ & $\begin{array}{l}-5.7 \\
-4.3\end{array}$ & $\begin{array}{l}-5.6 \\
-4.7\end{array}$ & $\begin{array}{l}-4.8 \\
-5.2\end{array}$ \\
\hline 666 & Soil & Italy & $\begin{array}{l}A \\
B\end{array}$ & + & $\begin{array}{l}- \\
+\end{array}$ & + & $\begin{array}{l}+ \\
+\end{array}$ & $\begin{array}{l}-5.2 \\
-6.8\end{array}$ & $\begin{array}{l}-7.0 \\
-6.3\end{array}$ & $\begin{array}{l}-1.0 \\
-5.2\end{array}$ & $\begin{array}{l}-4.8 \\
-4.9\end{array}$ \\
\hline 684 & Unknown & Unknown & $\begin{array}{l}A \\
B\end{array}$ & + & $\begin{array}{l}+ \\
+\end{array}$ & + & $\begin{array}{l}+ \\
+\end{array}$ & $\begin{array}{l}-6.1 \\
-4.9\end{array}$ & $\begin{array}{l}-4.5 \\
-5.1\end{array}$ & $\begin{array}{l}-4.4 \\
-4.0\end{array}$ & $\begin{array}{l}-4.4 \\
-4.8\end{array}$ \\
\hline 685 & Soil & Poland & $\begin{array}{l}A \\
B\end{array}$ & $\begin{array}{l}+ \\
+\end{array}$ & $\begin{array}{l}+ \\
+\end{array}$ & $\begin{array}{l}+ \\
+\end{array}$ & $\begin{array}{l}+ \\
+\end{array}$ & $\begin{array}{l}-5.1 \\
-4.7\end{array}$ & $\begin{array}{l}-4.8 \\
-4.5\end{array}$ & $\begin{array}{l}-4.3 \\
-4.3\end{array}$ & $\begin{array}{l}-4.7 \\
-4.0\end{array}$ \\
\hline 686 & Unknown & Poland & $\begin{array}{l}A \\
B\end{array}$ & $\begin{array}{l}+ \\
+\end{array}$ & $\begin{array}{l}+ \\
+\end{array}$ & $\begin{array}{l}+ \\
+\end{array}$ & $\begin{array}{l}+ \\
+\end{array}$ & $\begin{array}{l}-4.8 \\
-4.3\end{array}$ & $\begin{array}{l}-4.8 \\
-5.8\end{array}$ & $\begin{array}{l}-4.2 \\
-4.3\end{array}$ & $\begin{array}{l}-3.9 \\
-4.4\end{array}$ \\
\hline 687 & Soil & Poland & $\begin{array}{l}A \\
B\end{array}$ & $\begin{array}{l}+ \\
+\end{array}$ & $\begin{array}{l}+ \\
+\end{array}$ & $\begin{array}{l}+ \\
+\end{array}$ & $\begin{array}{l}+ \\
+\end{array}$ & $\begin{array}{l}-6.8 \\
-4.4\end{array}$ & $\begin{array}{l}-5.0 \\
-4.6\end{array}$ & $\begin{array}{l}-4.9 \\
-4.4\end{array}$ & $\begin{array}{l}-5.0 \\
-4.4\end{array}$ \\
\hline 688 & Soil & Poland & $\begin{array}{l}A \\
B\end{array}$ & - & - & - & - & - & $\begin{array}{l}-9.4 \\
-9.7\end{array}$ & $\frac{-9.4}{-}$ & $\overline{-9.4}$ \\
\hline 689 & Soil & Poland & $\begin{array}{l}A \\
B\end{array}$ & - & - & + & - & $\overline{-9.3}$ & $\begin{array}{l}-6.2 \\
-5.8\end{array}$ & $\begin{array}{l}-7.2 \\
-6.5\end{array}$ & $\begin{array}{l}-6.4 \\
-6.0\end{array}$ \\
\hline 690 & Soil & Poland & $\begin{array}{l}A \\
B\end{array}$ & - & - & - & - & $-\overline{10.0}$ & - & $\underline{-9.6}$ & $\begin{array}{l}-9.8 \\
-9.7\end{array}$ \\
\hline 691 & Soil & Poland & $\begin{array}{l}A \\
B\end{array}$ & $\begin{array}{l}- \\
-\end{array}$ & - & - & - & $\overline{-8.8}$ & $\begin{array}{l}-9.7 \\
-8.5\end{array}$ & $\begin{array}{l}-9.3 \\
-9.8\end{array}$ & $\begin{array}{l}-9.6 \\
-9.4\end{array}$ \\
\hline
\end{tabular}

a Collection from Station de recherches de Sainte-Foy (now Soils and Crops Research and Development Centre).

b At $-5^{\circ} \mathrm{C}$ as detected by the rapid tube nucleation test: + indicates freezing within 10 min (Ina+); - indicates no freezing (Ina).

c Temperature at which crystallization was triggered when the temperature of the tube contents was lowered from $0^{\circ} \mathrm{C}$ to $-10^{\circ} \mathrm{C}$ at a rate of $0.1^{\circ} \mathrm{C}$ min ${ }^{-1}$.

$\mathrm{d}$ - means that no freezing had crystallization occurred when the temperature reached $-10^{\circ} \mathrm{C}$. 
The present finding of ice-nucleating activity at warm temperatures in another species of Fusarium suggests the need to screen even more fungal isolates for ice nucleation, including species other than Fusarium, and suggests that ice nucleation activity plays an important role in either virulence or environmental compatibility and survival. The role of ice nucleation in fungi needs to be investigated further.

The method used here to detect INA was simple and efficient. It allows to determine quickly if a given culture is Ina ${ }^{+}$ or not. However, as opposed to the dropfreezing assay (Vali 1995), the rapid tube nucleation test can neither be used to quantify ice nuclei, nor to establish the cumulative ice nucleation spectrum. Pouleur et al. (1992) suggested the use of ice nucleation activity as an aid for the identification of Fusarium species. We know now that four fungal species are able to produce ice nuclei: $F$. acuminatum, $F$. avenaceum, $F$. oxysporum and F. tricinctum. This characteristic may prove useful in taxonomic treatment of these species.

\section{ACKNOWLEDGEMENTS}

We wish to thank C. Aubé, Agriculture and Agri-Food Canada, Saint-Hyacinthe, Quebec, Canada; A. Devaux, MAPAQ, Saint-Hyacinthe, Quebec, Canada; Canadian Collection of Fungal Cultures, Agriculture and Agri-Food Canada, Ottawa, Ontario, Canada; L. Gordon, Winnipeg, Manitoba, Canada; J.H. Graham, Silver Spring, Maryland, USA; K.T. Leath, USDA, University Park, Pennsylvania, USA; A. Logrieco, Instituto Tossine e Micotossine, Bari, Italy; B. Salleh, School of Biological Sciences, Pulau Pinang, Malaysia; and K. Seifert, Agriculture and Agri-Food Canada, Ottawa, Ontario, Canada, for providing Fusarium strains.

\section{REFERENCES}

Ashworth, E.N., and T.L. Kieft. 1995. Ice nucleation activity associated with plants and fungi. Pages 137-162 in R.E. Lee, G.J. Warren, and L.V. Gusta (eds.), Biological ice nucleation and its applications. APS Press, St. Paul, Minnesota.

Farr, D.F., G.F. Bills, G.P. Chamuris, and A.Y. Rossman. 1989. Fungi on plants and plant products in the United States. APS Press, St. Paul, Minnesota. 1252 pp.

Hasegawa, Y., Y. Ishihara, and T. Tokuyama. 1994. Characteristics of ice-nucleation activity in Fusarium avenaceum IFO 7158. Biosci. Biotech. Biochem. 58 : 2273-2274.

Hirano, S.S., E.A. Maher, A. Kelman, and C.D. Upper. 1978. Ice nucleation activity of fluorescent plant pathogenic pseudomonads. Pages 717-724 in Proc. $4^{\text {th }}$ Int. Conf. Plant Pathogenic Bacteria. Institut National de la Recherche Agronomique, Beaucozé, France.

Kieft, T.L. 1988. Ice nucleation activity in lichens. Appl. Environ. Microbiol. 54 : 16781681.

Paulin, J.-P., and J. Luisetti. 1978. Ice nucleation activity among phytopathogenic bacteria. Pages 725-731 in Proc. $4^{\text {th }}$ Int. Conf. Plant Pathogenic Bacteria. Institut National de la Recherche Agronomique, Beaucozé, France.

Phelps, P., T.H. Giddings, M. Prochoda, and R. Fall. 1986. Release of cell nuclei by Erwinia herbicola. J. Bacteriol. 167 : 496-502.

Pouleur, S., C. Richard, J.-G. Martin, and H. Antoun. 1992. Ice nucleation activity in Fusarium acuminatum and Fusarium avenaceum. Appl. Environ. Microbiol. 58 : 2960-2964.

Richard, C., C. Willemot, R. Michaud, and C. Gagnon. 1982. Low-temperature interactions in Fusarium wilt and root rot of alfalfa. Phytopathology 72 : 293-297.

Richard, C., R. Michaud, C. Willemot, M. Bernier-Cardou, and C. Gagnon. 1985. Effect of frost on Fusarium root rot of alfalfa and possibility of double-selection. Pages 209211 in C.A. Parker, A.D. Rovira, K.J. Moore, and P.T.W. Wong (eds.), Ecology and management of soilborne plant pathogens. Proc. of sect. 5 of the $4^{\text {th }}$ Int. Congr. of Plant Pathol. Melbourne, 1983. Am. Phytopathol. Soc., St. Paul, Minnesota. 
Smith M., and C.R. Olien. 1978. Pathological factors affecting survival of winter barley following controlled freeze test. Phytopathology 68 : 773-777.

Sychrova, E., J. Zamecnik, and J. Bieblova. 1994. Ice nucleation induced by spores and mycelium of Fusarium avenaceum and Fusarium tricinctum. Int. Union Microbiol. Soc. Congr., Prague, Czech Republic, July 3-8, 1994 (Abstract).

Tsumuki, H., H. Konno Maeda, and Y. Okamoto. 1992. An ice-nucleating active fungus isolated from the gut of the rice stem borer, Chilo suppressalis Walker [Lepidoptera : Pyralidae]. J. Insect Physiol. 38: 119-125.
Vali, G. 1995. Principles of ice nucleation. Pages 1-28 in R.E. Lee, G.J. Warren, and L.V. Gusta (eds.), Biological ice nucleation and its applications. APS Press, St. Paul, Minnesota.

Vali, G., M. Christensen, R.W. French, E.L. Gakian, L.R. Maki, and R.C. Schnell. 1976. Biogenic ice nuclei: Part II. Bacterial sources. J. Atmos. Sci. 33 : 1565-1570. 